\title{
Correlation and Path Coefficients Studies of Some Morphological and Seed Vigour Traits in Barley Cultivars (Hordeum vulgare L.) under Two Sowing Conditions
}

\author{
Suman Devi ${ }^{\text {** }}$, Yogender Kumar', Rakesh Kumar ${ }^{1}$, \\ Vijay Daneva ${ }^{2}, \operatorname{Paras}^{1}$ and Ram Nivas ${ }^{3}$
}

Department of Genetics and Plant Breeding, CCS Haryana Agricultural University, Hisar-125004 (Haryana), India

${ }^{2}$ Department of forestry, CCS Haryana Agricultural University, Hisar-125004

(Haryana), India

${ }^{3}$ Department of basic science \& humanities, CCS Haryana Agricultural University, Hisar-125004 (Haryana), India

*Corresponding author

\section{A B S T R A C T}

\section{Keywords}

Barley, Correlation,

Path coefficient,

Timely, Late sown

\section{Article Info}

Accepted:

17 September 2019

Available Online:

10 October 2019
The experimental material comprised of 50 diverse genotypes were sown in randomized block design with three replications in both timely and late sown condition to study correlation and path coefficient for ten quantitative field traits like days to heading, days to maturity, plant height $(\mathrm{cm})$, spike length $(\mathrm{cm})$, number of tillers per meter row, number of grains per spike, 1000 grain weight $(\mathrm{g})$, harvest index (\%), biological yield $(\mathrm{kg} / \mathrm{plot})$, grain yield $(\mathrm{kg} / \mathrm{plot})$ and eight seed parameters viz; seedling length $(\mathrm{cm})$, seed density $(\mathrm{g} / \mathrm{cc})$, standard germination (\%), seedling dry weight $(\mathrm{mg})$, vigour index I, vigour index II, electrical conductivity $(\mu \mathrm{S} / \mathrm{cm} / \mathrm{seed})$ and accelerated ageing at 48 and 72 hours. The grain yield was found to be positively and significantly associated with harvest index, 1000 grain weight and biological yield per plot in both the environments. Characters such as germination per cent, accelerated ageing $(48 \mathrm{~h}, 72 \mathrm{~h})$ and days to maturity were significantly and positively correlated with grain yield under timely sown condition likewise vigour index-I, seedling dry weight and number of tillers per meter were some other traits which had significant positive association with grain yield under late sown condition. Path coefficient analysis indicated that biological yield per plot, harvest index, vigour index-II and seedling length had high positive and significant direct effect in both the environments. Accordingly, emphasis could be given on these traits during selection for varietal improvement programme. 


\section{Introduction}

Barley is recognized as first domesticated Cereal and played an important role in the emergence of agriculture in the old world. It is stable food crop for millions of people in developing countries. By virtue of its nature, lower cost of cultivation, superior nutritional qualities, and better adaptability to harsh environments, barley is preferred by farmers as well as it has been considered, as poor man's crop. It is grown over wide range of environments, such as rainfed, irrigated, dry land, saline/alkaline soil, marginal lands, drought prone areas, hill regions and flood prone marginal/coastal areas in the world. Under stressful environments barley require less input while its adaptability is better in comparison to other cereals Owing to its hardiness. There is rich evidence of barley in the archaeological record from numerous sites throughout Near and Middle east, supporting the conception that it was a common and important crop in ancient times. Our ancestors were aware with the importance of crop and use of barley in religious ceremonies has been described in history. Among the cereal crops, barley ranks fourth in world cereal crop production and is mainly used as feed to livestock and poultry, human food (sattu, dalia and chapatti) and serves as a substrate for the production of alcoholic beverages, particularly beer, and it is probably the first drink developed by Neolithic human. Now Barley is key ingredient for beer and wine (whisky) industry. It has immense potential as quality cereal especially for nutrition and medicinal point of view. In European countries it is used as a breakfast food. Due to very low gluten, it is easily digestible as compared to wheat. It became as a successful crop, because of its short life cycle and morphological, physiological, and genetic characteristics. It is one of the important cereals of the world cultivated over an area of 575.00 lakh ha. The major barley growing countries are Russia,
China, Canada, USA, Spain, France, Australia, UK and India. At national level, barley is cultivated on area about 0.68 million ha area with total production of 1.79 million tons and productivity of $26.41 \mathrm{q} / \mathrm{ha}$ (ICARIIWBR, 2018). Among the major barley growing states, Rajasthan ranks first in the list of barley production $(0.81 \mathrm{mt})$ followed by Uttar Pradesh $(0.45 \mathrm{mt})$ and Madhya Pradesh $(0.26 \mathrm{mt})$. These three states altogether accounted for 85 per cent of the total national barley production. Haryana state achieved a production level of 73 thousand tons on 21,000 hectares. The average crop productivity in barley is highest in Punjab (3596 kg/ha) followed by Haryana (3476 $\mathrm{kg} / \mathrm{ha}$ ), Rajasthan (3046 kg/ha) and Uttar Pradesh (2774 kg/ha) (ICAR-IIWBR, 2018).

Barley is the main source of calories and improves micro nutrients, multi nutrition hormonal balance and treatment of many acute illnesses like blood pressure, osteoarthritis, gastric, ulcer, kidney stone and cancer. Barley foods have many health-enchaining attributes in addition to providing sound nutrition. As compared to wheat, Barley foods are beneficial in various ways and it also known as an diuretics, emollient used in case of pancreas and other digestive problems. In addition, barley is indispensible in virtually every Hindu ritual ceremony as sacred grain. Barley flourishes well under limited resources of irrigation and fertilizers. Barley has betaglucan and anticholesterol substance, acetyl choline which energies our nervous system and recover memory losses. It is easily digestible due to low gluten, soluble dietary fibres, high lysine, thiamine, and riboflavin which provide inflammatory effect.

In any breeding programme aiming at improving yield, it is essential to know, the degree of association between yield and other metric traits. Yield is complex trait, which is contributed by many independent traits and 
improvement in yield depends upon improvement in its component traits. Correlation coefficient ensures the absolute degree of association, genetic or non genetic relationship between two or more characters which forms the basis for selection. Wright, (1921) suggested path analysis which provides a clear understanding of the direct and indirect effect of various components attributing to the expression of grain yield. Path and correlation coefficient analyses are used widely to define the nature of complex interrelationships among yield components and to identify the sources of variation in yield. Knowledge derived on the extent and nature of interrelationship among characters helps in formulating efficient scheme of multiple trait selection in relation to agricultural practice. Therefore, an attempt was made to study these aspects in the present investigation to identify desirable characters for breeding programmes under two different sowing conditions viz; timely and late sown.

\section{Materials and Methods}

The experimental material for investigation was comprised of 50 diverse genotypes of barley grown under two different environments i.e. (i) timely and (ii) late sown and were evaluated using randomized block design (RBD) with three replications at Wheat and Barley section, Department of Genetics and Plant Breeding, Chaudhary Charan Singh, Haryana Agricultural University, Hisar during Rabi 2016-17 under irrigated conditions. The location of Hisar is on the outer margins of the South-West monsoon region with average annual rainfall of $450 \mathrm{~mm}$. during crop season of 2016-2017. Each genotype was grown in three rows with a plot size of $3.0 \times 0.69 \mathrm{~m}^{2}$ and the recommended cultural practices were adopted for raising healthy crop. Five competitive plants in each replication were randomly selected for recorded observation on 10 quantitative traits viz. days to heading, days to maturity, plant height $(\mathrm{cm})$, spike length (cm), number of tillers per meter row, number of grains per spike, 1000 grain weight $(\mathrm{g})$, harvest index $(\%)$, biological yield $(\mathrm{kg} / \mathrm{plot})$ and grain yield ( $\mathrm{kg} / \mathrm{plot})$ for all the traits under study except of days to heading, days to maturity, biological yield and grain yield which were recorded on plot basis. Average of the data from the sampled plant of each plot in respect to different traits was used for various statistical analyses. Further, the value of harvest index was calculated as per the formula given by Donald and Humblin (1976). Eight seed parameters viz; seedling length $(\mathrm{cm}), \quad$ seed density $(\mathrm{g} / \mathrm{cc})$, standard germination (\%), seedling dry weight $(\mathrm{mg})$, vigour index I, vigour index II, electrical conductivity $(\mu \mathrm{S} / \mathrm{cm} / \mathrm{seed})$ and accelerated ageing at 48 and 72 hours were also recorded to detect the vigour potential. Data recorded on the above characters were subjected to correlation coefficient analysis as suggested by Al-Jibouri et al., (1958). Its significance was tested by comparing at an appropriate level of significance of correlation coefficient at (n-2) degree of freedom, where ' $n$ ' was number of genotypes. Path coefficient analysis was carried out according to Dewey and Lu (1959).

\section{Results and Discussion}

\section{Correlation analysis}

The estimates of genotypic correlation coefficients among different characters are depicted in Table 1, 2, 3 (a) and 3 (b) for timely and late sown conditions. Genotypic correlation estimates under timely sown condition showed highly significant positive association of grain yield per plot with harvest index, biological yield, germination per cent, 1000 grain weight, accelerated ageing (48 h, $72 \mathrm{~h}$ ) and days to maturity while it was significant negatively associated with spike length, seedling length and seed density. Other 
characters such as plant height, number of grains per spike, seedling dry weight and vigour index-II had non-significant positive correlation with grain yield while days to heading, number of tillers per meter, vigour index-I and electrical conductivity were negatively associated with grain yield.

Under late sown condition, positive and highly significant correlation of grain yield was recorded with harvest index, biological yield, 1000 grain weight, vigour index-II, seedling dry weight and number of tillers per meter. Characters such as days to heading, days to maturity spike length, seedling length, vigour index-I, and seed density showed significant negative correlation with grain yield. Other traits such as plant height and accelerated ageing $(48 \mathrm{~h}, 72 \mathrm{~h})$ showed positive correlation with grain yield while number of grains per spike, germination per cent and electrical conductivity had negative correlation with grain yield.

Under both the growing environments, characters namely harvest index, 1000 grain weight and biological yield per plot showed significant positive association with grain yield while spike length, seed density and seedling length showed highly negative and significant correlation with grain yield. Some researchers also reported significant positive correlation of grain yield with harvest index (Drikvand et al., 2011and Kumar et al., 2013a), with 1000 grain weight (Kumar et al., 2013; Singh et al., 2014a and Singh et al., 2015b) and with biological yield per plot (Singh et al., 2014b and Shrimali et al., 2017). Drikvand et al., (2011) also found significant negative correlation of spike length with grain yield.

In both the environments, days to heading had highly significant positive correlation with days to maturity, plant height, spike length and number of grains per spike whereas it was negatively and significantly correlated with 1000 grain weight, harvest index, seedling dry weight, vigour index-II and electrical conductivity. Najeeb and Wani (2004) and Shrimali et al., (2017) also reported that days to heading had significant positive correlation with days to maturity. Singh et al., (2015b) concluded that days to heading had significant positive correlation with days to maturity, spike length and plant height. Days to maturity had found significant positive correlation with plant height and spike length in both the environments. Similar findings were also reported by Singh et al., (2015a). Characters such as number of grains per spike and biological yield per plot had significant positive association with days to maturity under timely sown and with accelerated ageing (48 h) under late sown condition. It was negatively and significantly related with 1000 grain weight, seed density, seedling dry weight and vigour index-II in both conditions. Positive correlation of days to maturity with number of grains per spike was also found by Singh et al., (2014a) and with biological yield per plot by Verma and Verma (2011). Number of tillers per meter had significant positive correlation with 1000 grain weight, biological yield per plot, seedling dry weight and vigour index-II under both environments. Positive association between number of tillers per meter and 1000 grain weight had also been reported by Kishor et al., (2000) and Singh et al., (2014a) and with biological yield per plot was observed by Singh et al., (2014b).

Number of grains per spike was positively and significantly correlated with harvest index and accelerated ageing ( $48 \mathrm{~h}, 72 \mathrm{~h}$ ) under timely sown and with germination per cent under late sown condition. Similar observation of positive association between number of grains per spike and harvest index was also reported by Yadav et al., (2014). Characters such as seedling dry weight and vigour index-II had significant negative correlation with number 
of grains per spike under both conditions. Biological yield per plot was positively and significantly correlated with germination per cent and vigour index-II under timely sown condition and the correlation was nonsignificant under late sown condition. Characters such as seedling length, seed density and vigour index-I had significant negative correlation with biological yield per plot under timely sown condition. Electrical conductivity under late sown condition also had significant negative correlation with biological yield per plot.

Harvest index was positively and significantly associated with germination per cent under timely sown condition while it was negatively correlated under late sown condition. Germination per cent had significant positive correlation with seedling length under late sown and negative under timely sown condition. Seedling dry weight was positively and significantly correlated with vigour indexI and vigour index-II under both conditions. Electrical conductivity was positively and significantly correlated with accelerated ageing (48 h, $72 \mathrm{~h})$ under timely sown condition while it was negatively and significantly correlated with accelerated ageing $(48 \mathrm{~h})$ under late sown condition. Accelerated ageing $(48 \mathrm{~h})$ had significant positive correlation with accelerated ageing $(72 \mathrm{~h})$ under both the environments. These results indicated that characters such as harvest index, 1000 grain weight and biological yield per plot were the major grain yield contributing traits under both the environments would be given more priority of selection pressure for improving grain yield in barley.

\section{Path analysis}

The vague of correlation coefficients provide association (positive or negative) between characters but it does not confirm causal basis of such associations. Path coefficient analysis gives the information on direct and indirect effects of various independent components on the dependent character. Direct and indirect effects of different characters on grain yield per plot were calculated under both environments which have been presented in Table 4 and 5. The positive and significant direct effect on grain yield was exerted by biological yield per plot, harvest index, seedling length and vigour index-II under both conditions. Verma (2011), Kumar et al., (2013) and Shrimali et al., (2017) also reported positive and significant direct effect of harvest index and biological yield on grain yield in barley.

Harvest index had high positive direct effect and it also contributed towards grain yield via number of grains per spike seedling length, germination per cent and vigour index-I under timely sown condition whereas under late sown condition, characters such as 1000 grain weight, seedling dry weight, vigour index-II, electrical conductivity and accelerated ageing (72 h) had high positive indirect effect on grain yield. The present finding, harvest index contributed indirectly via number of grains per spike was also reported by Singh et al., (2014b). Under both the environments biological yield per plot showed high positive and significant direct effect as well as accorded towards grain yield via days to maturity, plant height, number of tillers per meter, 1000 grain weight, germination per cent and accelerated ageing ( $48 \mathrm{~h}, 72 \mathrm{~h})$ under timely sown condition. Similarly, under late sown condition characters like plant height, number of tillers per meter, 1000 grain weight, germination per cent, seedling dry weight, vigour index-II and accelerated ageing (48 h) had positive indirect effect on grain yield. These findings were in consonance with the results of Singh et al., (2014b) for indirect positive effect of biological yield via number of tillers per meter. 
Table.1 Genotypic correlation coefficient between different morphological and seed vigour traits in barley genotypes under timely sown condition

\begin{tabular}{|c|c|c|c|c|c|c|c|c|c|c|c|c|c|c|c|c|c|c|c|}
\hline Characters & DH & DM & PH & SpL & T/M & G/S & $1000 \mathrm{GW}$ & BY/P & GY/P & HI & SL & SD & GP & SDW & VI & VII & EC & AA 48 & AA72 \\
\hline DH & & & & & & & & & & & & & & & & & & & \\
\hline DM & $0.795^{* *}$ & & & & & & & & & & & & & & & & & & \\
\hline PH & $0.292^{* *}$ & $0.303^{* *}$ & & & & & & & & & & & & & & & & & \\
\hline SpL & $0.207^{*}$ & $0.235^{* *}$ & 0.143 & & & & & & & & & & & & & & & & \\
\hline T/M & -0.058 & -0.045 & $-0.325^{* *}$ & $0.215^{* *}$ & & & & & & & & & & & & & & & \\
\hline G/S & $0.215^{* *}$ & $0.221^{* *}$ & $0.175^{*}$ & $-0.216^{* * *}$ & $-0.779^{* *}$ & & & & & & & & & & & & & & \\
\hline $1000 \mathrm{GW}$ & $-0.574^{* *}$ & $-0.314^{* *}$ & 0.035 & -0.092 & $0.398^{* *}$ & $-0.601^{* *}$ & & & & & & & & & & & & & \\
\hline BY/P & 0.062 & $0.362^{\text {** }}$ & $0.374^{* *}$ & -0.104 & $0.183^{*}$ & -0.085 & $0.310^{* * *}$ & & & & & & & & & & & & \\
\hline GY/P & -0.132 & $0.178^{*}$ & 0.115 & $-0.307^{* *}$ & -0.096 & 0.105 & $0.288^{* *}$ & $0.722^{* *}$ & & & & & & & & & & & \\
\hline HI & $-0.225^{* *}$ & -0.099 & $-0.259^{* *}$ & $-0.351^{* *}$ & $-0.315^{* *}$ & $0.224^{* *}$ & 0.065 & 0.071 & $0.732^{* *}$ & & & & & & & & & & \\
\hline SL & $-0.325^{* *}$ & $-0.321^{* * *}$ & 0.059 & 0.025 & $-0.208^{*}$ & 0.005 & 0.074 & $-0.453^{* *}$ & $-0.185^{*}$ & 0.156 & & & & & & & & & \\
\hline SD & 0.032 & $-0.172^{*}$ & 0.091 & 0.098 & $0.246^{* *}$ & $-0.335^{* *}$ & $0.237^{* *}$ & $-0.185^{*}$ & $-0.179^{*}$ & -0.110 & $0.268^{* *}$ & & & & & & & & \\
\hline GP & $-0.170^{*}$ & -0.036 & 0.151 & -0.055 & $0.286^{* *}$ & 0.052 & 0.062 & $0.616^{* *}$ & $0.609^{* *}$ & $0.338^{* * *}$ & $-0.394^{* *}$ & $-0.497^{* *}$ & & & & & & & \\
\hline SDW & $-0.627^{* *}$ & $-0.446^{* *}$ & 0.106 & 0.137 & $0.400^{* *}$ & $-0.627^{* *}$ & $0.832^{* *}$ & 0.156 & 0.093 & -0.052 & $0.336 *$ & $0.303^{* *}$ & $-0.172^{*}$ & & & & & & \\
\hline VI & $-0.325^{* *}$ & $-0.291^{* *}$ & 0.041 & 0.039 & $-0.209^{*}$ & 0.033 & 0.050 & $-0.407^{* *}$ & -0.148 & $0.169^{*}$ & $0.977^{* *}$ & $0.201^{*}$ & -0.045 & $0.302^{* *}$ & & & & & \\
\hline VII & $-0.633^{* *}$ & $-0.442^{* *}$ & 0.116 & 0.138 & $0.400^{* *}$ & $-0.617^{* *}$ & $0.835^{* *}$ & $0.170^{*}$ & 0.113 & -0.036 & $0.345^{* *}$ & $0.296^{* *}$ & -0.054 & $0.997^{* *}$ & $0.326^{* *}$ & & & & \\
\hline EC & -0.142 & $-0.181^{*}$ & $-0.366^{* *}$ & 0.037 & 0.056 & 0.050 & 0.069 & -0.066 & -0.064 & 0.011 & $0.231^{* *}$ & -0.081 & $0.240^{* *}$ & 0.047 & $0.241^{* *}$ & 0.064 & & & \\
\hline AA48 & -0.031 & -0.010 & $0.197^{*}$ & 0.030 & 0.002 & $0.183^{*}$ & 0.103 & $0.381^{* *}$ & $0.265^{* *}$ & 0.015 & 0.005 & $-0.269^{* *}$ & $1.164^{* *}$ & 0.019 & 0.088 & 0.057 & $0.334^{* *}$ & & \\
\hline AA72 & 0.001 & 0.054 & 0.152 & 0.091 & -0.069 & $0.312^{* *}$ & -0.059 & $0.287^{* *}$ & $0.284^{* *}$ & 0.119 & -0.032 & $-0.214^{* *}$ & $1.235^{* *}$ & -0.153 & 0.065 & -0.114 & $0.249^{* *}$ & $0.838^{* * *}$ & \\
\hline
\end{tabular}

** Significant at $1 \%, *$ Significant at $5 \%$ 


\section{Int.J.Curr.Microbiol.App.Sci (2019) 8(10): 2210-2222}

Table.2 Genotypic correlation coefficient between different morphological and seed vigour traits in barley genotypes under late sown condition

\begin{tabular}{|c|c|c|c|c|c|c|c|c|c|c|c|c|c|c|c|c|c|c|c|}
\hline Characters & DH & DM & PH & SpL & $\mathbf{T} / \mathbf{M}$ & G/S & $1000 \mathrm{GW}$ & BY/P & GY/P & HI & SL & SD & GP & SDW & VI & VII & EC & AA48 & AA72 \\
\hline \multicolumn{20}{|l|}{ DH } \\
\hline DM & $0.821^{* *}$ & & & & & & & & & & & & & & & & & & \\
\hline PH & $0.434^{* *}$ & $0.304^{* *}$ & & & & & & & & & & & & & & & & & \\
\hline SpL & $0.625^{* *}$ & $0.564^{* *}$ & $0.239^{* *}$ & & & & & & & & & & & & & & & & \\
\hline $\mathbf{T} / \mathbf{M}$ & -0.066 & 0.060 & -0.020 & 0.025 & & & & & & & & & & & & & & & \\
\hline G/S & $0.182^{*}$ & 0.103 & $0.334^{* *}$ & -0.055 & $0.601^{* *}$ & & & & & & & & & & & & & & \\
\hline $1000 \mathrm{GW}$ & $-0.615^{* *}$ & $-0.457^{* *}$ & $-0.379^{* * *}$ & $-0.341^{* *}$ & $0.165^{*}$ & $-0.372^{* *}$ & & & & & & & & & & & & & \\
\hline BY/P & 0.110 & 0.103 & $0.601^{* *}$ & -0.069 & $0.441^{* *}$ & 0.159 & 0.096 & & & & & & & & & & & & \\
\hline GY/P & $-0.438^{* *}$ & $-0.319^{* *}$ & 0.068 & $-0.456^{* *}$ & $0.217^{* *}$ & -0.004 & $0.553^{* *}$ & $0.634^{* *}$ & & & & & & & & & & & \\
\hline HI & $-0.675^{* *}$ & $-0.527^{* *}$ & $-0.410^{* *}$ & $-0.580^{* *}$ & -0.051 & $-0.192^{*}$ & $0.661^{* *}$ & -0.021 & $0.760^{* *}$ & & & & & & & & & & \\
\hline SL & -0.056 & -0.084 & -0.105 & $0.196^{*}$ & -0.063 & -0.093 & -0.059 & $-0.366^{* *}$ & $-0.341^{* *}$ & -0.114 & & & & & & & & & \\
\hline SD & -0.157 & $-0.179^{*}$ & -0.039 & $0.166^{*}$ & $-0.220^{* *}$ & -0.111 & 0.066 & $-0.223^{* *}$ & $-0.188^{*}$ & -0.097 & $0.218^{* *}$ & & & & & & & & \\
\hline GP & 0.056 & -0.141 & $0.201^{*}$ & 0.071 & -0.140 & $0.243^{* *}$ & $-0.403^{* *}$ & 0.038 & -0.114 & $-0.200^{*}$ & $0.524^{* *}$ & 0.080 & & & & & & & \\
\hline SDW & $-0.579^{* *}$ & $-0.366^{* *}$ & $-0.217^{* *}$ & $-0.201^{*}$ & $0.228^{* *}$ & $-0.360^{* *}$ & $0.779^{* *}$ & 0.094 & $0.412^{* *}$ & $0.489^{* *}$ & $0.334^{* *}$ & 0.101 & -0.031 & & & & & & \\
\hline VI & -0.036 & -0.094 & -0.047 & $0.194^{*}$ & -0.081 & -0.028 & -0.131 & $-0.324^{* *}$ & $-0.324^{* *}$ & -0.135 & $0.989^{* *}$ & $0.197^{*}$ & $0.645^{* *}$ & $0.284^{* *}$ & & & & & \\
\hline VII & $-0.577^{* * *}$ & $-0.398^{* *}$ & $-0.162^{*}$ & $-0.194^{*}$ & $0.209^{*}$ & $-0.320^{* * *}$ & $0.728^{* *}$ & 0.118 & $0.414^{* *}$ & $0.472^{* *}$ & $0.391^{* *}$ & 0.102 & 0.100 & $0.993^{* * *}$ & $0.357^{* *}$ & & & & \\
\hline EC & $-0.284^{* *}$ & -0.005 & $-0.471^{* *}$ & -0.076 & -0.076 & $-0.238^{* *}$ & $0.498^{* *}$ & $-0.418^{* *}$ & -0.077 & $0.274^{* *}$ & $0.209^{*}$ & 0.113 & $-0.203^{*}$ & $0.504^{* *}$ & 0.147 & $0.463^{* *}$ & & & \\
\hline AA48 & $0.188^{*}$ & $0.170^{*}$ & $0.337^{* *}$ & 0.001 & $-0.251^{* *}$ & $0.255^{* *}$ & -0.041 & 0.126 & 0.006 & -0.099 & -0.040 & -0.057 & -0.063 & -0.016 & -0.059 & -0.036 & $-0.258^{* *}$ & & \\
\hline AA72 & -0.112 & -0.140 & -0.037 & -0.090 & $-0.302^{* *}$ & 0.123 & $0.209^{*}$ & -0.145 & 0.098 & $0.243^{* *}$ & -0.011 & 0.036 & $-0.212^{* *}$ & 0.096 & -0.049 & 0.068 & 0.006 & $0.696^{* *}$ & \\
\hline
\end{tabular}

** Significant at $1 \%, *$ Significant at $5 \%$ 
Table.3(a) Correlation coefficients (positive) among different characters under timely and late sown conditions

\begin{tabular}{|c|c|c|c|}
\hline \multirow[t]{2}{*}{ Characters } & \multirow{2}{*}{$\begin{array}{c}\text { Type of } \\
\text { correlation }\end{array}$} & \multicolumn{2}{|c|}{ Environments } \\
\hline & & Timely sown & Late sown \\
\hline DH & + ve & $\mathrm{DM}^{* *}, \mathrm{PH}^{* *}, \mathrm{SpL}^{* *}, \mathrm{G} / \mathrm{S} * *, \mathrm{BY} / \mathrm{P}, \mathrm{SD}, \mathrm{AA} 72$ & $\mathrm{DM}^{* *}, \mathrm{PH}^{* *}, \mathrm{SpL}^{*}, \mathrm{G} / \mathrm{S} *, \mathrm{BY} / \mathrm{P}, \mathrm{GP}, \mathrm{AA} 48^{*}$ \\
\hline DM & +ve & $\mathrm{PH}^{* *}, \mathrm{SpL}^{* *}, \mathrm{G} / \mathrm{S}^{* *}, \mathrm{BY} / \mathrm{P}^{* *}, \mathrm{GY} / \mathrm{P}^{*}, \mathrm{AA} 72$ & $\mathrm{PH}^{* *}, \mathrm{SpL}{ }^{* *}, \mathrm{~T} / \mathrm{M}, \mathrm{G} / \mathrm{S}, \mathrm{BY} / \mathrm{P}, \mathrm{AA} 48^{*}$ \\
\hline PH & $+\mathrm{ve}$ & $\begin{array}{c}\text { SpL,G/S*, 1000GW, BY/P**, GY/P,SL, SD, GP, SDW, } \\
\text { VI, VII, AA48*, AA72 }\end{array}$ & $\mathrm{SpL} * *, \mathrm{G} / \mathrm{S} * *, \mathrm{BY} / \mathrm{P} * *, \mathrm{GY} / \mathrm{P}, \mathrm{GP} *, \mathrm{AA} 48^{* *}$ \\
\hline SpL & + ve & T/M**, SL, SD, SDW, VI, VII, EC, AA48, AA72 & $\mathrm{T} / \mathrm{M}, \mathrm{SL}^{*}, \mathrm{SD} *, \mathrm{GP}, \mathrm{VI}^{*}, \mathrm{AA} 48^{*}$ \\
\hline $\mathbf{T} / \mathbf{M}$ & + ve & $\begin{array}{c}1000 \mathrm{GW}^{* *}, \mathrm{BY} / \mathrm{P}^{*}, \mathrm{SD}^{* *}, \mathrm{GP}^{* *}, \mathrm{SDW}^{* *}, \mathrm{VII} * *, \mathrm{EC}, \\
\mathrm{AA} 48\end{array}$ & G/S**, 1000GW*, BY/P**, GY/P**, SDW**, VII* \\
\hline G/S & + ve & GY/P, HI**, SL, GP, VI, EC, AA48*, AA72** & $\mathrm{BY} / \mathrm{P}, \mathrm{GP} * *, \mathrm{AA} 48^{* *}, \mathrm{AA} 72$ \\
\hline $1000 \mathrm{GW}$ & + ve & $\begin{array}{c}\mathrm{BY} / \mathrm{P}^{* *}, \mathrm{GY} / \mathrm{P} * *, \mathrm{HI}, \mathrm{SL}, \mathrm{SD}^{* *}, \mathrm{GP}, \mathrm{SDW} * *, \mathrm{VI}, \mathrm{VII}, \\
\mathrm{EC}, \mathrm{AA} 48\end{array}$ & $\mathrm{BY} / \mathrm{P}, \mathrm{GY} / \mathrm{P}^{* *}, \mathrm{HI}{ }^{* *}, \mathrm{SD}, \mathrm{SDW} * *, \mathrm{VII} * *, \mathrm{EC}^{* *}, \mathrm{AA} 72^{*}$ \\
\hline BY/P & +ve & GY/P**, HI, GP**, SDW, VII*, AA48**, AA72** & GY/P**, GP, SDW, VII, AA48 \\
\hline GY/P & +ve & HI**, GP**, SDW, VII, AA48**, AA72** & $\mathrm{HI}^{* *}, \mathrm{SDW}^{* *}, \mathrm{VII} * *, \mathrm{AA} 48, \mathrm{AA} 72$ \\
\hline HI & +ve & $\mathrm{SL}, \mathrm{GP}^{* *}, \mathrm{VI} *, \mathrm{EC}, \mathrm{AA} 48, \mathrm{AA} 72$ & $\mathrm{SDW}^{* *}, \mathrm{VII} * *, \mathrm{EC}^{* *}, \mathrm{AA} 72 * *$ \\
\hline $\mathbf{S L}$ & +ve & $\mathrm{SD}^{* *}, \mathrm{SDW}^{* *}, \mathrm{VI}{ }^{* *}, \mathrm{VII}{ }^{* *}, \mathrm{EC}^{* *}, \mathrm{AA} 48$ & $\mathrm{SD}^{* *}, \mathrm{GP}^{* *}, \mathrm{SDW}^{* *}, \mathrm{VI}^{* *}, \mathrm{VII}^{* *}, \mathrm{EC}^{*}$ \\
\hline SD & + ve & $\mathrm{SDW}^{* *}, \mathrm{VI} *, \mathrm{VII} * *$ & GP,SDW, VI*, VII, EC, AA72 \\
\hline GP & +ve & $\mathrm{EC} * *, \mathrm{AA} 48 * *, \mathrm{AA} 72 * *$ & VI**, VII \\
\hline SDW & + ve & $\mathrm{VI}^{* *}, \mathrm{VII} * *, \mathrm{EC}, \mathrm{AA} 48$ & $\mathrm{VI}^{* *}, \mathrm{VII} * *, \mathrm{EC}^{* *}, \mathrm{AA} 72$ \\
\hline VI & + ve & VII**, EC**, AA48, AA72 & VII**, EC \\
\hline VII & +ve & EC, AA48 & $\mathrm{EC}^{* *}, \mathrm{AA} 72$ \\
\hline EC & +ve & AA48**, AA72** & AA72 \\
\hline AA48 & +ve & $\mathrm{AA} 72 * *$ & $\mathrm{AA} 72 * *$ \\
\hline AA72 & +ve & ...... & $\ldots \ldots$ \\
\hline
\end{tabular}


Table.3(b) Correlation coefficients (negative) among different characters under timely and late sown conditions

\begin{tabular}{|c|c|c|c|}
\hline \multirow[t]{2}{*}{ Characters } & \multirow{2}{*}{$\begin{array}{l}\text { Type of } \\
\text { correlation }\end{array}$} & \multicolumn{2}{|c|}{ Environments } \\
\hline & & Timely sown & Late sown \\
\hline DH & -ve & $\begin{array}{l}\mathrm{T} / \mathrm{M}, 1000 \mathrm{GW}^{* *}, \mathrm{GY} / \mathrm{P}^{* *}, \mathrm{HI}^{* *}, \mathrm{SL}^{* *}, \mathrm{GP}^{* *} \\
\mathrm{SDW}^{* *}, \mathrm{VI}^{* *}, \mathrm{VII}^{* *}, \mathrm{EC}, \mathrm{AA} 48\end{array}$ & $\begin{array}{c}\mathrm{T} / \mathrm{M}, 1000 \mathrm{GW}^{* *}, \mathrm{HI}^{* *}, \mathrm{SL}, \mathrm{SD}, \mathrm{SDW}^{* *}, \mathrm{VI}, \mathrm{VII} * *, \mathrm{EC}^{* *}, \\
\text { AA72 }\end{array}$ \\
\hline DM & -ve & $\begin{array}{c}\mathrm{T} / \mathrm{M}, 1000 \mathrm{GW}^{* *}, \mathrm{HI}, \mathrm{SL}^{* *}, \mathrm{SD}^{*}, \mathrm{GP}, \mathrm{SDW}^{* *}, \\
\mathrm{VI}^{* *}, \mathrm{VII} * * \\
\mathrm{EC} *, \mathrm{AA} 48\end{array}$ & $\begin{array}{c}1000 \mathrm{GW}^{* *}, \mathrm{GY} / \mathrm{P} * *, \mathrm{HI}^{* *}, \mathrm{SL}, \mathrm{SD} *, \mathrm{GP}, \mathrm{SDW}^{*}, \mathrm{VI}, \\
\text { VII**, EC, AA72 }\end{array}$ \\
\hline PH & -ve & $\mathrm{T} / \mathrm{M}^{* *}, \mathrm{HI}^{* *}, \mathrm{EC}^{* *}$ & $\begin{array}{l}\text { T/M, } 1000 \mathrm{GW}^{* *}, \mathrm{HI}^{* *}, \mathrm{SL}, \mathrm{SD}, \mathrm{SDW}^{* *}, \mathrm{VI}, \mathrm{VII}^{*}, \mathrm{EC}^{* *}, \\
\mathrm{AA} 72\end{array}$ \\
\hline SpL & -ve & $\mathrm{G} / \mathrm{S} * *, 1000 \mathrm{GW}, \mathrm{BY} / \mathrm{P}, \mathrm{GY} / \mathrm{P}^{* *}, \mathrm{HI}{ }^{* *}, \mathrm{GP}$ & $\begin{array}{c}\mathrm{G} / \mathrm{S}, 1000 \mathrm{GW}^{* *}, \mathrm{BY} / \mathrm{P}, \mathrm{GY} / \mathrm{P}^{* *}, \mathrm{HI}^{* *}, \mathrm{SDW}^{*}, \mathrm{VII}^{*}, \mathrm{EC}, \\
\text { AA72 }\end{array}$ \\
\hline $\mathbf{T} / \mathbf{M}$ & -ve & G/S**, GY/P, HI**, SL*, VI*, AA72 & HI, SL, SD**, GP, VI, EC, AA48**, AA72** \\
\hline $\mathbf{G} / \mathbf{S}$ & -ve & $1000 \mathrm{GW}, \mathrm{BY} / \mathrm{P}, \mathrm{SD}^{* *}, \mathrm{SDW}^{* *}, \mathrm{VII} * *$ & $1000 \mathrm{GW}^{* *}, \mathrm{GY} / \mathrm{P}, \mathrm{HI}^{*}, \mathrm{SL}, \mathrm{SD}, \mathrm{SDW}^{* *}, \mathrm{VI}, \mathrm{VII} * *, \mathrm{EC}^{* *}$ \\
\hline $1000 \mathrm{GW}$ & -ve & AA72 & SL, GP**, VI, AA48 \\
\hline BY/P & -ve & $\mathrm{SL}^{* *}, \mathrm{SD}^{*}, \mathrm{VI} * *, \mathrm{EC}$ & $\mathrm{HI}, \mathrm{SL}^{* *}, \mathrm{SD}^{* *}, \mathrm{VI} * *, \mathrm{EC} * *, \mathrm{AA} 72$ \\
\hline GY/P & -ve & $\mathrm{SL}^{*}, \mathrm{SD}^{*}, \mathrm{VI}, \mathrm{EC}$ & $\mathrm{SL}^{* *}, \mathrm{SD}^{*}, \mathrm{GP}, \mathrm{VI} * *, \mathrm{EC}$ \\
\hline HI & -ve & SD, SDW, VII & SL, SD, GP*, VI, AA48 \\
\hline SL & -ve & GP**, AA72 & AA48, AA72 \\
\hline SD & -ve & $\mathrm{GP} * *, \mathrm{EC}, \mathrm{AA} 48 * *, \mathrm{AA} 72 * *$ & AA48 \\
\hline GP & -ve & $\mathrm{SDW}^{*}, \mathrm{VI}, \mathrm{VII}$ & $\mathrm{SDW}, \mathrm{EC} *, \mathrm{AA} 48, \mathrm{AA} 72 * *$ \\
\hline SDW & -ve & AA72 & AA48 \\
\hline VI & -ve & $\ldots .$. & AA48, AA72 \\
\hline VII & -ve & AA72 & AA48 \\
\hline EC & -ve & $\ldots \ldots$ & AA48** \\
\hline AA48 & -ve & $\ldots \ldots$ & $\ldots \ldots$ \\
\hline AA72 & -ve & $\ldots \ldots$ & $\ldots \ldots$ \\
\hline
\end{tabular}


Table.4 Direct (diagonal) and indirect effects of different characters on grain yield in barley under timely sown condition

\begin{tabular}{|c|c|c|c|c|c|c|c|c|c|c|c|c|c|c|c|c|c|c|c|}
\hline Characters & DH & DM & PH & SpL & $\mathbf{T} / \mathbf{M}$ & G/S & $\begin{array}{l}1000 \\
\text { GW }\end{array}$ & BY/P & HI & SL & SD & GP & SDW & VI & VII & EC & AA48 & AA72 & $\begin{array}{c}\text { rg with } \\
\text { grain } \\
\text { yield } \\
\text { (g/plot) }\end{array}$ \\
\hline DH & -0.005 & -0.004 & -0.002 & -0.001 & 0.000 & -0.001 & 0.003 & 0.000 & 0.001 & 0.002 & 0.000 & 0.001 & 0.003 & 0.002 & 0.003 & 0.001 & 0.000 & 0.000 & -0.132 \\
\hline DM & -0.004 & -0.005 & -0.002 & -0.001 & 0.000 & -0.001 & 0.002 & -0.002 & 0.001 & 0.002 & 0.001 & 0.000 & 0.002 & 0.001 & 0.002 & 0.001 & 0.000 & 0.000 & $0.178^{*}$ \\
\hline PH & 0.004 & 0.005 & 0.015 & 0.002 & -0.005 & 0.003 & 0.001 & 0.006 & -0.004 & 0.001 & 0.001 & 0.002 & 0.002 & 0.001 & 0.002 & -0.006 & 0.003 & 0.002 & 0.115 \\
\hline SpL & 0.006 & 0.007 & 0.004 & 0.028 & 0.006 & -0.006 & -0.003 & -0.003 & -0.010 & 0.001 & 0.003 & -0.002 & 0.004 & 0.001 & 0.004 & 0.001 & 0.001 & 0.003 & $-0.307^{* *}$ \\
\hline $\mathbf{T} / \mathbf{M}$ & -0.003 & -0.002 & -0.016 & 0.011 & 0.050 & -0.039 & 0.020 & 0.009 & -0.016 & -0.010 & 0.012 & 0.014 & 0.020 & -0.011 & 0.020 & 0.003 & 0.000 & -0.003 & -0.096 \\
\hline G/S & 0.021 & 0.022 & 0.017 & -0.021 & -0.076 & 0.097 & -0.059 & -0.008 & 0.022 & 0.000 & -0.033 & 0.005 & -0.061 & 0.003 & -0.060 & 0.005 & 0.018 & 0.030 & 0.105 \\
\hline $1000 \mathrm{GW}$ & -0.070 & -0.038 & 0.004 & -0.011 & 0.049 & -0.074 & 0.123 & 0.038 & 0.008 & 0.009 & 0.029 & 0.008 & 0.102 & 0.006 & 0.102 & 0.008 & 0.013 & -0.007 & $0.288^{* *}$ \\
\hline BY/P & 0.041 & 0.241 & 0.249 & -0.069 & 0.122 & -0.057 & 0.207 & 0.667 & 0.047 & -0.302 & -0.123 & 0.411 & 0.104 & -0.271 & 0.113 & -0.044 & 0.254 & 0.191 & $0.722^{* *}$ \\
\hline HI & -0.149 & -0.066 & -0.172 & -0.233 & -0.209 & 0.148 & 0.043 & 0.047 & 0.663 & 0.103 & -0.073 & 0.224 & -0.034 & 0.112 & -0.024 & 0.007 & 0.010 & 0.079 & $0.732^{* *}$ \\
\hline SL & -0.095 & -0.095 & 0.017 & 0.007 & -0.061 & 0.001 & 0.022 & -0.133 & 0.046 & 0.294 & 0.079 & -0.116 & 0.099 & 0.287 & 0.102 & 0.068 & 0.001 & -0.009 & $-0.185^{*}$ \\
\hline SD & 0.000 & 0.002 & -0.001 & -0.001 & -0.002 & 0.003 & -0.002 & 0.002 & 0.001 & -0.003 & -0.010 & 0.005 & -0.003 & -0.002 & -0.003 & 0.001 & 0.003 & 0.002 & $-0.179^{*}$ \\
\hline GP & -0.001 & 0.000 & 0.001 & 0.000 & 0.002 & 0.000 & 0.001 & 0.005 & 0.003 & -0.003 & -0.004 & 0.008 & -0.001 & 0.000 & 0.000 & 0.002 & 0.009 & 0.010 & $0.609^{* *}$ \\
\hline SDW & 0.353 & 0.251 & -0.060 & -0.077 & -0.225 & 0.353 & -0.469 & -0.088 & 0.029 & -0.189 & -0.170 & 0.097 & -0.563 & -0.170 & -0.561 & -0.027 & -0.011 & 0.086 & 0.093 \\
\hline VI & 0.084 & 0.075 & -0.011 & -0.010 & 0.054 & -0.008 & -0.013 & 0.105 & -0.044 & -0.253 & -0.052 & 0.012 & -0.078 & -0.259 & -0.084 & -0.062 & -0.023 & -0.017 & -0.148 \\
\hline VII & -0.321 & -0.224 & 0.059 & 0.070 & 0.203 & -0.313 & 0.424 & 0.086 & -0.018 & 0.175 & 0.150 & -0.028 & 0.506 & 0.165 & 0.507 & 0.033 & 0.029 & -0.058 & 0.113 \\
\hline EC & 0.006 & 0.008 & 0.016 & -0.002 & -0.003 & -0.002 & -0.003 & 0.003 & 0.000 & -0.010 & 0.004 & -0.011 & -0.002 & -0.011 & -0.003 & -0.045 & -0.015 & -0.011 & -0.064 \\
\hline AA48 & 0.002 & 0.001 & -0.011 & -0.002 & 0.000 & -0.010 & -0.006 & -0.021 & -0.001 & 0.000 & 0.015 & -0.064 & -0.001 & -0.005 & -0.003 & -0.018 & -0.055 & -0.046 & $0.265^{* *}$ \\
\hline AA72 & 0.000 & 0.002 & 0.005 & 0.003 & -0.002 & 0.010 & -0.002 & 0.009 & 0.004 & -0.001 & -0.007 & 0.041 & -0.005 & 0.002 & -0.004 & 0.008 & 0.028 & $\mathbf{0 . 0 3 3}$ & $0.284^{* *}$ \\
\hline
\end{tabular}

Residual effect: 0.006; $\mathrm{rg}=$ genotypic correlation; *, ** Significant at 0.05 and 0.01 level, respectively 
Table.5 Direct (diagonal) and indirect effects of different characters on grain yield in barley under late sown condition

\begin{tabular}{|c|c|c|c|c|c|c|c|c|c|c|c|c|c|c|c|c|c|c|c|}
\hline Characters & DH & DM & PH & SpL & $\mathbf{T} / \mathbf{M}$ & G/S & $1000 \mathrm{GW}$ & BY/P & HI & SL & SD & GP & SDW & VI & VII & EC & AA48 & AA72 & $\begin{array}{c}\text { rg with } \\
\text { grain } \\
\text { yield } \\
\text { (g/plot) }\end{array}$ \\
\hline DH & -0.053 & -0.044 & -0.023 & -0.033 & 0.004 & -0.010 & 0.033 & -0.006 & 0.036 & 0.003 & 0.008 & -0.003 & 0.031 & 0.002 & 0.031 & 0.015 & -0.010 & 0.006 & $-0.438^{* *}$ \\
\hline DM & 0.096 & 0.118 & 0.036 & 0.066 & 0.007 & 0.012 & -0.054 & 0.012 & -0.062 & -0.010 & -0.021 & -0.017 & -0.043 & -0.011 & -0.047 & -0.001 & 0.020 & -0.017 & $-0.319^{* *}$ \\
\hline PH & -0.008 & -0.006 & -0.019 & -0.004 & 0.000 & -0.006 & 0.007 & -0.011 & 0.008 & 0.002 & 0.001 & -0.004 & 0.004 & 0.001 & 0.003 & 0.009 & -0.006 & 0.001 & 0.068 \\
\hline SpL & 0.039 & 0.035 & 0.015 & 0.062 & 0.002 & -0.003 & -0.021 & -0.004 & -0.036 & 0.012 & 0.010 & 0.004 & -0.013 & 0.012 & -0.012 & -0.005 & 0.000 & -0.006 & $-0.456^{* *}$ \\
\hline $\mathbf{T} / \mathbf{M}$ & -0.005 & 0.005 & -0.002 & 0.002 & 0.078 & -0.047 & 0.013 & 0.034 & -0.004 & -0.005 & -0.017 & -0.011 & 0.018 & -0.006 & 0.016 & -0.006 & -0.020 & -0.024 & $0.217^{* *}$ \\
\hline G/S & 0.020 & 0.012 & 0.038 & -0.006 & -0.068 & 0.113 & -0.042 & 0.018 & -0.022 & -0.010 & -0.013 & 0.027 & -0.041 & -0.003 & -0.036 & -0.027 & 0.029 & 0.014 & -0.004 \\
\hline $1000 \mathrm{GW}$ & -0.052 & -0.039 & -0.032 & -0.029 & 0.014 & -0.031 & 0.085 & 0.008 & 0.056 & -0.005 & 0.006 & -0.034 & 0.066 & -0.011 & 0.062 & 0.042 & -0.003 & 0.018 & $0.553^{* * *}$ \\
\hline BY/P & 0.062 & 0.058 & 0.338 & -0.039 & 0.248 & 0.090 & 0.054 & 0.562 & -0.012 & -0.206 & -0.125 & 0.021 & 0.053 & -0.182 & 0.066 & -0.235 & 0.071 & -0.082 & $0.634^{* *}$ \\
\hline HI & -0.569 & -0.445 & -0.345 & -0.489 & -0.043 & -0.162 & 0.557 & -0.018 & 0.843 & -0.096 & -0.082 & -0.169 & 0.412 & -0.114 & 0.398 & 0.231 & -0.083 & 0.205 & $0.760^{* *}$ \\
\hline SL & -0.027 & -0.040 & -0.050 & 0.093 & -0.030 & -0.044 & -0.028 & -0.174 & -0.054 & 0.476 & 0.104 & 0.249 & 0.159 & 0.470 & 0.186 & 0.099 & -0.019 & -0.005 & $-0.341^{* *}$ \\
\hline SD & -0.009 & -0.010 & -0.002 & 0.010 & -0.013 & -0.006 & 0.004 & -0.013 & -0.006 & 0.013 & 0.057 & 0.005 & 0.006 & 0.011 & 0.006 & 0.006 & -0.003 & 0.002 & $-0.188^{*}$ \\
\hline GP & 0.006 & -0.015 & 0.021 & 0.007 & -0.014 & 0.025 & -0.042 & 0.004 & -0.021 & 0.054 & 0.008 & 0.104 & -0.003 & 0.067 & 0.010 & -0.021 & -0.006 & -0.022 & -0.114 \\
\hline SDW & 0.349 & 0.220 & 0.131 & 0.121 & -0.137 & 0.217 & -0.469 & -0.056 & -0.294 & -0.201 & -0.061 & 0.019 & -0.602 & -0.171 & -0.598 & -0.303 & 0.010 & -0.058 & $0.412^{* *}$ \\
\hline VI & 0.021 & 0.055 & 0.027 & -0.113 & 0.047 & 0.016 & 0.076 & 0.188 & 0.079 & -0.575 & -0.115 & -0.375 & -0.165 & -0.582 & -0.207 & -0.085 & 0.035 & 0.029 & $-0.324^{* *}$ \\
\hline VII & -0.325 & -0.224 & -0.091 & -0.109 & 0.118 & -0.180 & 0.410 & 0.066 & 0.266 & 0.220 & 0.057 & 0.056 & 0.559 & 0.201 & 0.563 & 0.261 & -0.020 & 0.038 & $0.414^{* *}$ \\
\hline EC & 0.017 & 0.000 & 0.028 & 0.004 & 0.004 & 0.014 & -0.029 & 0.024 & -0.016 & -0.012 & -0.007 & 0.012 & -0.030 & -0.009 & -0.027 & -0.059 & 0.015 & 0.000 & -0.077 \\
\hline AA48 & 0.000 & 0.000 & 0.000 & 0.000 & 0.000 & 0.000 & 0.000 & 0.000 & 0.000 & 0.000 & 0.000 & 0.000 & 0.000 & 0.000 & 0.000 & 0.000 & 0.000 & 0.000 & 0.006 \\
\hline AA72 & 0.000 & 0.000 & 0.000 & 0.000 & 0.000 & 0.000 & 0.000 & 0.000 & 0.000 & 0.000 & 0.000 & 0.000 & 0.000 & 0.000 & 0.000 & 0.000 & -0.001 & -0.002 & 0.098 \\
\hline
\end{tabular}

Residual effect: $0.0010 ; \mathrm{rg}=$ genotypic correlation; *, ** Significant at 0.05 and 0.01 level, respectively 
1000 grain weight showed positive direct effect and also contributed towards grain yield via seedling dry weight, vigour index-II, number of tillers per meter and biological yield under timely sown and harvest index, seedling dry weight, vigour index-II and electrical conductivity under late sown condition. 1000 grain weight contributed through biological yield was also reported by Yadav et al., (2014). Positive direct effect of 1000 grain weight on grain yield was also confirmed by Singh et al., (2014a) and Singh et al., (2015a). Germination per cent showed low positive direct effect on grain yield and it also contributed positively towards grain yield via biological yield, harvest index, number of tillers per meter, electrical conductivity and accelerated ageing (48 h, $72 \mathrm{~h}$ ) under timely sown, whereas, under late sown condition characters like plant height, number of grains per spike, seedling length, vigour index-I and vigour index-IIhad positive indirect effect on grain yield. This indicates that traits like harvest index, biological yield per plot and 1000 grain weight had high positive direct and indirect effect on grain yield.

The low residual effects under both the environments indicated that most of the variability in the genotypes for the characters under study had been explained by the independent variables included in the analysis, which was also supported by Singh et al., (2014b).

This study had demonstrated that the grain yield of barley has significant and positive correlations with harvest index, 1000 grain weight and biological yield per plot under both sown conditions. These relations meant that any increase in any one of the yield components would be caused some increase in grain yield. Path analysis revealed that different yield components had different effects on grain yield. Finally, it was concluded that biological yield per plot, harvest index, vigour index-II and seedling length had stronger positive effects on grain yield than did the other components. The results of this study indicate that harvest index, 1000 grain weight and biological yield may be used as selection criteria for new cultivars of barley with higher grain yield.

\section{References}

Al-Jibouri, H.A., Miller, P.A. and Robinson, H.F. (1958). Genotypic and environmental variances and covariances in an upland cotton cross of interspecific origin. Agronomy Journal, 50: 633-636.

Dewey, D.R. and Lu, K.H. (1959). A correlation and path coefficient analysis of component of crested wheat grass seed production. Agronomy Journal, 51: 515-518.

Drikvand, R., Samiei, K. and Hossinpor, T. (2011). Path coefficient analysis in hull-less barley under rainfed condition. Australian Journal of Basic and Applied Sciences, 5 (12): 277-279.

Donald, C. M., and J. Humblin, (1976). The Biological Yield and Harvest Index of Cereals as Agronomic and Plant Breeding Criteria. Advances in Agronomy, $28: 361-405$.

ICAR-IIWBR (2018). Director's Report of AICRP on Wheat and Barley 20172018, Ed: G.P. Singh, ICAR-Indian Institute of Wheat and Barley Research, Karnal, Haryana, India. P 94.

Kishor R., Pandey, D.D. and Verma, S.K. (2000). Genetic variability and character association in hull-less barley (Hordeum vulgare L.). Crop Research, 19 (2): 241-244.

Kumar, Y., Lamba, R.A.S., Verma, S.R. and Niwas, R. (2013). Genetic variability for yield and its components in Barley 
(Hordeum vulgare L.). Forage Research, 39 (2): 67-70.

Najeeb, S. and Wani, S. A. (2004). Correlation and path analysis studies in barley (Hordeum vulgare L.). National Journal of Plant Improvement, 6 (2): $124-125$.

Singh, A., Seth, V. and Goswami, A. (2015b). Study of genetic parameters for yield and yield contributing trait of elite genotypes of barley (Hordeum vulgare L.). Trends in Biosciences, 8 (4): 898901.

Singh, J., Prasad, L.C., Madakemohekar, A.H. and Bornare, S.S. (2014a). Genetic variability and character association in diverse genotypes of barley (Hordeum vulgare L.). The Bioscan, 9 (2): 759761.

Singh, S., Madakemohekar, A.H., Prasad, L.C. and Prasad, R. (2015a). Genetic variability and correlation analysis of yield and its contributing traits in barley (Hordeum vulgare L.) for drought tolerance. Indian Research Journal of Genetics and Biotechnology, 7 (1): 103-108.
Singh, S.K., Verma, P.N., Singh, L., Ali, T. and Prasad, K.D. (2014b). Variability and divergence analysis in barley $(H$. vulgare L.) under irrigated condition. Trends in Biosciences, 7(6): 452-456.

Verma, I. and Verma, S.R. (2011). Genotypic variability and correlations among morpho-physiological traits affecting grain yield in barley (Hordeum vulgare L.). Journal of Wheat Research, 3 (1): 37-42.

Verma, Indresh (2011). Genotypic variability and association analysis for grain yield and its attributes in timely and late sown barley (Hordeum vulgare L.). M.Sc. Thesis, CCS Haryana Agricultural University.

Yadav, S.K., Pawar, K.K., Baghel, S.S., Jarman, M. and Singh, A.K. (2014). Genetic analysis for grain yield and its components in barley (Hordeum vulgare L.). Journal of Wheat Research, 6 (2): 163-166.

Wright, S. (1921). Correlation and causation. Journal of Agricultural Research. 20: 557-585.

\section{How to cite this article:}

Suman Devi, Yogender Kumar, Rakesh Kumar,Vijay Daneva, Paras and Ram Nivas. 2019. Correlation and Path Coefficients Studies of Some Morphological and Seed Vigour Traits in Barley Cultivars (Hordeum vulgare L.) under Two Sowing Conditions. Int.J.Curr.Microbiol.App.Sci. 8(10): 2210-2222. doi: https://doi.org/10.20546/ijcmas.2019.810.257 